\title{
Probe! - Praxislabors für kreative Lernwege. Ein Konzept für offene Lernprozesse in heterogenen Lerngruppen
}

\author{
Gisela Fasse
}

\begin{abstract}
Zusammenfassung
Der vorliegende Artikel stellt das Format von „PROBE! - Praxislabors für kreative Lernwege" vor, das ausgehend von Projekten im vielsprachigen Subsahara-Afrika entwickelt wurde. Beispielhaft wird eines dieser Projekte beschrieben. Erfahrene Pädagogen bilden die „PROBE!“-Teams und führen in Workshops - den Praxislabors - Theater- und Spracharbeit zusammen. In heterogenen Lerngruppen nutzen die „Probe!“-Teams die Kreativität von Kindern und Jugendlichen in künstlerischen Arbeitsprozessen. Theatrale Verfahren werden dabei verbunden mit Ansätzen aus der Mehrsprachigkeitsdidaktik, mit Körperarbeit und Kommunikationstraining. Den Teilnehmer/innen wird so die Gelegenheit gegeben, ihre sprachliche und kulturelle Identität zu erforschen, sie wechselseitig anzuerkennen und eigene sprachliche, soziale und arbeitstechnische Kompetenzen weiter auszubilden. Alle Workshops schließen mit einer Präsentation $\mathrm{ab}$, die jedoch nicht das alleinige Ziel ist: vielmehr intensivieren die Teilnehmer/innen im Arbeitsprozess ihre Fähigkeit des präzisen Beobachtens, sie entwickeln eigene Notationssysteme und können so ihre Sicht auf Arbeitsverfahren und Ergebnis formulieren. Auf der Grundlage des jeweils eigenen Zugangs werden Inhalte aus Spracharbeit, Literatur und individuellen Lebensgeschichten der Teilnehmenden aus einer neuen Perspektive sichtbar und erfolgreich bearbeitet.
\end{abstract}

\section{Heterogenität als Chance}

Kinder, Jugendliche, Erwachsene erlernen (Fremd-)Sprachen nicht nur im dafür konzipierten Unterricht, sondern sie erwerben Kompetenzen immer auch informell und jedes Individuum verfügt über Dialekte und/oder Soziolekte. Insbesondere in Ländern Afrikas und Asiens ist Mehrsprachigkeit Normalität, ${ }^{1}$

\footnotetext{
${ }^{1}$ Südafrika hat allein elf offizielle Landessprachen, auch im Nachbarland Namibia werden neben der offiziellen Landessprache Englisch afrikanische Sprachen gesprochen. Dazu das folgende Zitat von Unotjari, einer 13jährigen Schülerin der DHPS in Windhoek: „Otjiherero ist meine erste Sprache. Wir sprechen diese Sprache in meiner Familie. Als ich fünf Jahre alt war, kam ich in den Kindergarten. Da redete niemand Otjiherero mit mir, sondern nur Englisch und Afrikaans. Am Anfang war das schwierig für mich, aber dann habe ich gelernt, die beiden Sprachen zu unterscheiden und zu sprechen... Seit dem 5. Schuljahr lerne ich jetzt Deutsch.“
} 
europäische Nationen sind Einwanderungsländer, in Deutschland wird längst nicht mehr nur Deutsch gesprochen. Städtische Schulen verzeichnen Schüler/innen aus verschiedensten Herkunftsländern, bis zu 70\% der Schülerschaft haben eine Migrationsgeschichte. Die „PROBE!“-Teams wollen die Heterogenität der Lerngruppen nutzen, denn diese Vielfalt ist eine Chance für alle Teilnehmerinnen und Teilnehmer in einer Gruppe. In den Praxislabors wird der Raum für Fragen, Beiträge und Kommentare geschaffen, die gerade im Kontext der Heterogenität, der Verschiedenheit von Kulturen und Perspektiven unvorhergesehene, aber bedeutsame Wahrnehmungs- und Lernprozesse initiieren können. So gab zum Beispiel nach einer Vorstellungsrunde in einer mehrsprachigen Gruppe deutscher und südafrikanischer Jugendlicher die Frage eines deutschsprachigen Teilnehmers nach der Aussprache und Bedeutung eines afrikanischen Vornamens Anlass zu Freude bei dem gefragten Mädchen: sie hatte selten die Erfahrung gemacht, dass man sich dafür interessierte. Gleichzeitig wurde dies zum Ausgangspunkt einer Reflexion: welche Bedeutung haben unsere Namen für uns persönlich und unsere Umwelt?

Gerade Kinder und Jugendliche profitieren von einem breiten Angebot möglicher Lernwege. In ihrer Verschiedenheit lernen sie unterschiedlich schnell und nehmen Angebote unterschiedlich auf. Die „PROBE!“-Workshops bieten solche Lernmöglichkeiten. Die Teilnehmer/innen können aus einem breiten Angebot das für sie jeweils Passende auswählen und in einer Struktur des kooperativen Lernens können sie ihr Potenzial einbringen. Aus der heterogenen Gruppe, deren Mitglieder sich zu Beginn fremd gegenüberstehen, wird im Verlauf des kreativen Prozesses ein Ensemble.

Warum dann: „PROBE!“ als Titel für diese Arbeitsform? Probe - das heißt: Prüfung, Untersuchung, Beweisverfahren; Bewährung(sversuch); Muster, Teststück, Probeaufführung.

Der Begriff ist programmatisch, denn er stellt heraus, dass etwas Geplantes im Entstehungsprozess ist, und dass auf einer bereits bestehenden Grundlage weiter untersucht, probiert, verändert wird. Im Vordergrund steht damit der Prozess, in dem das gemeinsame Betrachten, Entwickeln, Verändern und erneute Probieren der eigentliche Inhalt sind. Kritische und verändernde Fragen und Vorschläge erfahren so im Bewusstsein der Lernenden eine Aufwertung. Daraus ergibt sich auch unsere Bezeichnung der Workshops als Praxislabors. Teams aus Theaterpädagoginnen und -pädagogen und Lehrenden setzen in ihrer Projektplanung bei Wünschen und Bedürfnissen der jeweiligen (Lern)gruppe an. Davon ausgehend werden historische, literarische, biographische, naturwissenschaftliche Fragestellungen bearbeitet. Die Projektleiter/innen stellen ein reichhaltiges Angebot an Materialien, Übungen und Aufgaben bereit. Drama- und theaterpädagogische Übungen sind Bestandteil aller Projekte von „Probe! Praxislabors für kreative Lernwege“. Sie sollen den Teilnehmer/innen Formen des individuellen Ausdrucks, des gemeinsamen spielerischen Entwickelns und Darstellens bieten. 


\section{Gestaltung und Verfahren der Praxislabors}

Themen und Inhalte der Praxislabors sind vielfältig. Wesentlich ist die vielperspektivische Herangehensweise an die Thematik - so bezog sich etwa in der Internationalen Akademie The Climate Is Changing ${ }^{2}$ der Begriff des Klimas nicht nur auf die Meteorologie, sondern auch auf das soziale Klima und eröffnete den Teilnehmer/innen ein weites Feld für eigene Zugriffe auf das Thema.

Sprachen verändern sich, treten in Beziehung zueinander. In südafrikanischen Workshops war erlebbar, wie sich ausgehend vom Thema Sprache organisch Fragestellungen zu Geschichte und Geographie der jeweiligen Herkunftsländer entwickeln: etwa nach der Bedeutung der Einwanderung aus Europa nach Südafrika für die heutige Sprachenverbreitung des Afrikaans, Englischen und Deutschen; in Europa die Verwandtschaft innerhalb der germanischen und romanischen Sprachfamilien. Bezüge sowohl zum muttersprachlichen wie zum Unterricht in der/den Fremdsprache/n sind auf jeweils unterschiedliche Weise in den heterogenen Lerngruppen vorhanden und herstellbar.

Die Arbeitsweise der „PROBE!“-Teams basiert dabei stets auf Ansätzen und Verfahren aus folgenden Bereichen:

\subsection{Szenische Verfahren}

Je nach Aufgabenstellung regen Materialien wie Fotos, Bilder, Musiktitel oder Texte verschiedenster Art den kreativen Gruppenprozess an. In der eigentlichen Arbeit bedienen sich die Projektleiter/innen in der Regel szenischer Verfahren. ${ }^{3}$

Szenische Spielaufgaben ermutigen die Lernenden, im Schutz einer Rolle oder in der Obhut des Theaterchors ihr eigenes verbales und nonverbales Ausdrucksrepertoire zu entdecken, zu erweitern und sich mitSelbstvertrauen vor einem Publikum zu präsentieren. Sprachenvielfalt und individuell verschiedene Körperjargons, Gesten und Haltungen werden in der szenischen Arbeit nicht als trennende Grenze zwischen den Teilnehmerinnen und Teilnehmern gewertet, sondern als expressiver Fundus aller, der das Ausdrucksrepertoire der ganzen Gruppe erweitert. Dem einzelnen Lernenden wird Raum gegeben, sich im Als-ob des Theaters in verschiedenen Rollen auszuprobieren. Dieses Experimentieren mit Rollen fördert nicht nur die Persönlichkeitsentwicklung der Jugendlichen. Figurenarbeit gewährt den Schülern den nötigen Raum und gibt ihnen Ausdrucksformen an die Hand, mit denen sie ihre Anliegen, Themen, Träume, Ängste usw. kommunizieren können. Die szenischen Präsentationen sind sinnlich wahrnehmbare Vorgänge, an denen die Teilnehmenden der Werkstatt die Kunst des präzisen Zuschauens üben können. Dabei schärfen sie ihr Beobachtungsvermögen nicht nur im Bezug auf theatrale Mittel (stage drama), auch Rollenverhalten und Gesprächskonstellationen in der eigenen Lebenswelt (social drama) lassen sich erfassen und analysieren.

\footnotetext{
2 Siehe http://ww.ev-bildungszentrum.de/index.php?article_id=2\&SHOPLANG= deutsch\&SHOPKEY=monatsuebersicht/april/181_internationale+akademie+the+climate+ istchanging.html - 6.1.2012.

3 Zu Verfahren und Übungen im Einzelnen: Holl 2011.
} 


\subsection{Kommunikationstraining}

Die Arbeitsprozesse sind so angelegt, dass alle Gruppenmitglieder Verantwortung für das Lösen von Aufgabenstellungen übernehmen und gemeinsam ein Ergebnis erarbeiten. Die Projektleiter/innen führen Übungen ein, in denen die Schülerinnen und Schüler bestimmte Kommunikationskompetenzen ausbilden. Dazu gehören vor allem präzises Adressieren von Gesprächspartnern, aufmerksames Zuhören, Lesen des Gegenübers, spontanes und angemessenes Reagieren im Gespräch und bewusstes Einsetzen von Körpersprache.

\subsection{Körperarbeit}

Entspannungsübungen und dynamische Bewegungsspiele tragen dazu bei, Blockaden und Spannungen in der Lerngruppe abzubauen und fördern Offenheit und Aufnahmebereitschaft.

\section{Erfahrungen aus der Praxis: ,Literatur und Geschichte - und wir'}

Am Beispiel des im Jahr 2010 in Südafrika durchgeführten Projekts Literatur und Geschichte - und wir möchte ich darstellen, wie fruchtbar ein Arbeitsprozess im Sinn der Praxislabors sich entwickeln kann, in dessen Verlauf Jugendliche eigene Lernwege beschreiten. 22 Jugendliche aus sechs verschiedenen Schulen Südafrikas kamen für drei Tage in einem Camp zusammen, begleitet von je einer Lehrkraft jeder Schule. Diesen Schulen gemeinsam ist, dass an ihnen das Fach Deutsch unterrichtet wird und dass sie „Partnerschulen der Bundesrepublik Deutschland“, PASCH-Schulen, sind. Das Fach Deutsch als Fremdsprache gehört in das Curriculum der Schulen - allerdings in sehr verschiedener Ausprägung. An den drei Deutschen Auslandsschulen (DAS) Kapstadt, Johannesburg und Pretoria hat das Fach zentrale Bedeutung, während es an den staatlichen Schulen (hier der Pretoria High School for Girls, der Diamantveld High School aus Kimberley und der Wartburg-Kirchdorf High School aus KwaZulu-Natal) um sein Überleben kämpft. Für die Lernenden und die Lehrkräfte der Schulen hatte daher die Möglichkeit von Kooperation und Vernetzung besondere Bedeutung. Die Heterogenität der Jugendlichen, die in einem abgelegenen „Retreat" bei Johannesburg zusammen kamen, war groß: zufällig hätten sich die Teilnehmer/innen sicher nie getroffen. Die räumlichen Distanzen zwischen den Schulen betragen bis zu 2000 Kilometern; die Erstsprachen der Jugendlichen waren Zulu, Xhosa, Englisch, Afrikaans und Deutsch, soziale und kulturelle Herkunft und Hautfarbe waren verschieden. Nicht zu unterschätzen sind auch die Unterschiede in Schulorganisation und Unterrichtsformen, in Lern- und Arbeitsweise, die Schüler/innen und Lehrer/innen erleben. In der Planung des Projekts mussten diese Voraussetzungen berücksichtigt werden.

Als Projektleiterin konnte ich bei der Konzeption des Projekts auf Wünschen und Interessen von Lehrkräften und Schüler/innen aufbauen, die im Vorjahr an 
einem ähnlichen Projekt beteiligt gewesen waren. Gleichzeitig war mir möglich, das inhaltliche und methodische Angebot aufgrund meiner Erfahrungen an verschiedenen Auslandsschulen auszuweiten. ${ }^{4}$

\subsection{Komplexe Aufgabenstellungen}

Ziel des Projekts war es, die Kompetenzen in Verständnis und Anwendung der deutschen Sprache zu erweitern, Interesse an der deutschen und südafrikanischen Geschichte zu fördern, die Schulen untereinander zu vernetzen, den Jugendlichen in der noch immer gesellschaftlich divergenten Gesellschaft Südafrikas Raum zu geben, in dem Begegnung, Kennenlernen und Austausch ermöglicht und gefördert wird. Das Material- und Aufgabenangebot sollte die Lernenden in die Lage versetzen, durch gemeinsames Ausprobieren eine Präsentation zu entwickeln, eine Szene, einen Dialog, eine Installation, einen neuen Text. Mit dem thematischen Untertitel des Projekts: 1990 - 2010. 20 Jahre nach der Freilassung Nelson Mandelas und der deutschen Wiedervereinigung ist die Lebenszeit der Jugendlichen angesprochen, die Geschichte und Gegenwart ihrer Familie.

Für unser Vorhaben standen uns drei Tage zur Verfügung. Nicht alle Schritte konnten und sollten im Einzelnen vorausgeplant werden. Entscheidend für den Prozess der Zusammen- und Weiterarbeit war die Frage, ob und wie sich das Interesse der Jugendlichen an der anspruchsvollen Thematik entwickeln würde. Auf dieser Grundlage wäre dann ein eigenes, kreatives Arbeiten in Gruppen möglich. Ich konnte davon ausgehen, dass alle Anwesenden Freude an der deutschen Sprache und am Lesen hatten. Das historische Thema war neu. Das Schulfach „Geschichte“ ist für viele Jugendliche abstrakt; in Südafrika wird es an vielen Schulen wegen der geringen Nachfrage gar nicht unterrichtet. Gleichzeitig ist von großer Bedeutung, welche Rolle die Erwachsenen der Jahre vor 1990 gesellschaftlich gespielt haben. „Madiba“, Nelson Mandela, ist das große Vorbild. Die deutsche Geschichte gehört in den Bereich „Landeskunde“ des Deutschunterrichts.

$\mathrm{Zu}$ den Grundgedanken der Praxislabors gehört der Ansatz an der Individualität der Schülerinnen und Schüler. Wo liegt ihr Interesse, aus dem sich dann ein eigenständiges, erfolgreiches Arbeiten entwickeln kann? Das Interesse an der Geschichte würde ausgehen von der eigenen Lebenswelt und den Erzählungen in der Familie. Gleichzeitig erwartete ich Neugier aufgrund des Zusammentreffens verschiedener Familiengeschichten in der Gruppe.

\subsection{Kooperativer Arbeitsprozess}

Das anfängliche Warm-up und weitere Übungen, verbal und non-verbal, mit Musik und mit vorgegebenen Textbausteinen, ließen anfängliche Scheu

\footnotetext{
${ }^{4}$ Finanziert wurde das Projekt aus Mitteln der PASCH-Initiative durch die Zentralstelle für das Auslandsschulwesen, in deren Auftrag ich in den Jahren 2008-2011 als Fachberaterin für das Fach Deutsch an das südafrikanische Erziehungsministerium abgeordnet war.
} 
schwinden. Bewegungen wurden offener, weiter, der Raum wurde in seinen ganzen Ausmaßen genutzt. Bereits in dieser Phase wurde erkennbar, dass ritualisierte Verhaltensweisen aus den Kulturen als solche wahrgenommen und verstanden werden müssen, sollen keine Missverständnisse entstehen: so gilt es etwa in einigen afrikanischen Kontexten als respektlos, dem Gegenüber ins Gesicht zu schauen, während es in der europäischen Kultur gerade umgekehrt ist.

In den verschiedenen Übungen und Mini-Szenen wechselten die Jugendlichen immer wieder ihre Mitspieler, ihre Gruppe, so dass sich bald alle Teilnehmenden mit Namen kennen gelernt hatten. Aus dem spielerischen Umgang mit den verschiedenen Sprachen der Jugendlichen ergab sich ein Blick auf den Reichtum der Mehrsprachigkeit im Raum, der Hemmungen gegenüber der Zielsprache Deutsch relativieren konnte.

Zwei Mal setzten wir nach ,bewegtem' Beginn mit einer ,Frage des Tages ${ }^{5}$ ein. Aus den Antworten und der Reaktion auf die Frage Welchen Bezug hat die Geschichte deines Landes zur Geschichte deiner Familie? am dritten Tag unseres Zusammenseins wurde deutlich, dass es einen Sprung im Prozess der bewussten Auseinandersetzung mit der Thematik ebenso wie in der vertrauensvollen Zusammenarbeit gegeben hatte: die Jugendlichen erzählten so offen von Erinnerungen und Ereignissen aus ihren Familienbiographien, dass Nachfragen, Kommentare und Gespräche darüber sich in der Gruppe entwickelten. Durchaus dervon Unterdrückung und Gewalt geprägten Geschichte der Apartheid bewusst sagte ein Mädchen: „Ich bin stolz auf meine Vorfahren, die als Buren eine Farm aufgebaut haben, aber ich habe Angst darüber zu sprechen, weil man mich dann für eine Rassistin hält.“ Aus Deutschland stammende Jugendliche berichteten von Ausreise und Flucht aus der DDR, von ,Rosinenbombern' und Einwanderung nach Südafrika; südafrikanische Schüler/innen sprachen über die Zwänge der Passgesetze, der ,Homelands' und der unzulänglichen Bantu Education. Daraus ergaben sich in der Gruppe aber keine Barrieren, keine Zuschreibungen alter Muster, sondern es kam zu einer ernsthaften gegenseitigen Wahrnehmung. ${ }^{6}$

Gemeinsame Sprache während des Projekts war Deutsch - aber nicht ausschließlich und ausschließend. Abzuwägen war in diesem Zusammenhang, inwieweit sich eine Festschreibung des alleinigen Gebrauchs der Zielsprache Deutsch auf den sprachlichen Lernzuwachs einerseits, auf Spontaneität, Freude und Kooperationsbereitschaft andererseits auswirken würde. Reichten die sprachlichen Mittel in der deutschen Sprache nicht, dann stellten die Jugendlichen ihre inhaltlichen Beiträge also anders dar: mit Hilfe von Körpersprache oder auch in englischer Sprache. Einzelne Begriffe und Ausdrücke

\footnotetext{
5 Jede/r Teilnehmer/in erhält ein Kärtchen, auf das eine Antwort auf die zentral ausgehängte Frage geschrieben wird. Diese Karten werden ebenfalls aufgehängt und vorgelesen. So erhält jede/r Schüler/in die Gelegenheit vorbereitet zu sprechen; die Aufgabe der Projektleiter besteht in der Moderation des anschließenden Gesprächs.

${ }^{6}$ Auf die unsichere Nachfrage von Christine, sie wisse nie genau, ob sie von ,schwarzen' und ,weißen' Menschen reden dürfe, reagierte Nizo humorvoll: „With my friends I talk about raisins and peanuts instead of saying this one is white and this one is black." Und Busiswe ergänzte: "That's a silly problem except when your parents force it upon you."
} 
nannten die Schüler/innen auch in ihren Erstsprachen, aus denen sich Gespräche über die besondere Aussage und Bedeutung ergaben. Mit einem Beispiel möchte ich dies illustrieren: der Begriff ,dompas' ist die afrikaanssprachige Bezeichnung für den Pass, den alle Schwarzen und Farbigen unter den Apartheidsgesetzen tragen mussten. Das aus dem Wort ,dom' und dem Nomen ,pas' zusammengesetzte Wort lässt sich verschieden interpretieren: als dummer Pass oder als Pass für Dumme. Daraus ergab sich die Frage, warum sich dies afrikaanssprachige Wort, nicht aber eine Bezeichnung aus einer afrikanischen Sprache durchgesetzt hat. In solchen Gesprächen kann sich Sprachbewusstheit entwickeln und Reflexion über die Bezüge zwischen Sprache und Lebenswelt stattfinden.

Die abschließenden Präsentationen erfolgten in deutscher Sprache.

Die Textbasis für die Präsentationen waren Auszüge aus Jugendromanen, die ihre Erzählungen in der jüngeren deutschen und südafrikanischen Geschichte ansiedeln. Vielfältige weitere Materialien standen zur Verfügung: Karten, Lexika, Zeitschriften; zwei Spielfilme ${ }^{7}$ illustrierten die Thematik.

Die Gruppen von je drei Schüler/innen arbeiteten auf der Grundlage ,ihrer' Texte aus Jugendromanen und mit Gedichten. ${ }^{8}$ Zu den jeweiligen Texten waren zwei offene Aufgabenstellungen zur Auswahl gegeben, die wiederum modifiziert werden konnten. Alle Gruppen waren sprachlich heterogen zusammengesetzt. Einige Jugendliche waren deutschsprachig, andere hatten erst seit zwei Jahren Deutschunterricht.

Alle Kleingruppen arbeiteten selbstständig, steuerten ihre individuellen Arbeitsprozesse, erbaten Beratung nur in Ausnahmefällen von den anwesenden Lehrkräften. Die Gruppen arbeiteten in Sichtweite voneinander, sie motivierten sich so gegenseitig, konnten gegenseitige Hilfestellung oder Kritik erbitten. Eine Werkstattatmosphäre entstand. Die Jugendlichen schrieben eindrucksvolle eigene Geschichten und Szenen und setzten diese in eine Präsentation um.

In diesem Prozess des praktischen Entwickelns einer gemeinsamen Formensprache, in assoziativen Beiträgen und bildlichen Vorschlägen traten immer unterschiedliche Auffassungen, Lesarten und Haltungen zu den Inhalten zu Tage. Beim Ausprobieren mussten die Schüler/innen konkret verhandeln, wie ihre Präsentationen aussehen sollten. In ihrem gemeinsamen Probenprozess machten sie sich die Lerninhalte zu eigen.

Darüber hinaus war zu beobachten, wie die Schüler/innen in ihren Beschreibungen der eigenen Arbeit und der Präsentation von Zwischenergebnissen der andern Gruppen präziser wurden. Sie übten sich in solchen Arbeitsprozessen darin, alltägliche und ästhetische Phänomene mit großer Aufmerksamkeit wahrzunehmen, zu analysieren und dabei individuelle Kriterien für jeweilige Darbietungen zu entwickeln. Die Teilnehmenden erfuhren, dass sie dann

\footnotetext{
${ }^{7}$ Anne C. Voorhoeve, Lilly unter den Linden, Lingua Video; Skin, www. skinthemovie. net 6.1.2012

8 Acht Gruppen hatten neun Texte zur Auswahl, darunter zwei Gedichte der südafrikanischen Autorin Lebogang Mashile aus deren zweisprachig veröffentlichter Sammlung Töchter von morgen - Gedichte, Heidelberg: Verlag DasWunderhorn 2010.
} 
erfolgreich waren, wenn sie solidarisch zusammen arbeiteten, sich zuhörten, Anregungen aufgriffen, konstruktiv Kritik äußerten, Konflikte gemeinsam bearbeiteten. So wurde ein Schritt hin zum kooperativen, selbstverantwortlichen Lernen getan.

In den Ergebnissen der Projektarbeit zeigte sich, dass die Arbeiten zur Geschichte ihres Heimatlandes Südafrika und der Geschichte des Landes, dessen Sprache im Zentrum des Projekts stand, nicht bei einem reinen Nebeneinander stehen geblieben waren. Vielmehr hatten die Schüler/innen Erscheinungsformen von Trennung, Zwang und Aufbegehren in den historischen Prozessen beider Länder erkannt, die sie verglichen, analysierten und zum Thema ihrer Präsentationen machten. ${ }^{9}$

Komplex waren die Aufgabenstellungen, da die Schüler/innen aufgefordert waren:

- Bezüge zwischen biographischen Erfahrungen und historischen Fakten und Entwicklungen herzustellen;

- Verbindungslinien zu ziehen zwischen Literatur und eigenen Erfahrungen;

- Historische Entwicklungen in zwei Ländern - Deutschland und Südafrika - zu betrachten und zu vergleichen;

- Eine Umsetzung in Formen einer Präsentation zu leisten;

- In einer Fremdsprache zu kommunizieren;

- Sprache als Kommunikationsmittel zu reflektieren und zu thematisieren.

Die Kommentare und das Feedback der zuschauenden Gruppe nach den Abschlusspräsentationen waren präzise und konstruktiv. Einige theatrale Mittel wie Freeze,Verlangsamung, Spiegelung und Gedankenschatten hatten wir in unseren Übungen zuvor erprobt. Sie alle wurden in den Präsentationen bewusst eingesetzt und in ihrer Funktion und Aussage von den Zuschauern erkannt.

Unser Projekt schloss mit einer Evaluation durch die Schüler/innen und Lehrer/innen ab. Die Lehrkräfte waren sowohl selbst Teilnehmende in allen Phasen des Projekts als auch Beratende für die Schülergruppen gewesen. Sie wurden außerdem zusammen mit der Projektleiterin in einen Prozess der begleitenden Evaluation und Steuerung einbezogen.

\footnotetext{
9 Das Projekt und seine Ergebnisse sind dokumentiert. Eine pdf-Version ist eingestellt unter: http://www.auslandsschulwesen.de/cln_100/nn_2167452/Auslandsschulwesen/ Auslandsschularbeit/Fachberater/Afrika/Pretoria/Aktuelles/2011/inhalt_ _LiteraturGeschichte.html - 6.1.2012 Siehe auch: http://www.kreative-lernwege.com 6.1.2012
} 


\section{Ziele und Ergebnisse des Projekts}

Eine Erweiterung der Kompetenz in der Fremdsprache Deutsch kann in einem so kurzen Zeitraum nicht wirklich gemessen werden. Innerhalb der drei Projekttage aber war sehr deutlich geworden, dass die Teilnehmer/innen sich mit mehr Spontaneität und wachsendem Selbstbewusstsein in deutscher Sprache einbrachten. Die Schüler/innen setzten Körpersprache ein, sie umschrieben, fragten nach einzelnen Begriffen, wenn sie ihren Satz einmal nicht wie geplant mit deutschem Vokabular beenden konnten. Ebenso hatten sie gelernt, dass aufmerksames Zuhören und Zusehen ihnen helfen konnte, Bedeutungen zu erraten, Zusammenhänge zu erkennen: Fertigkeiten, die ihnen auch weiterhin beim Lernen und im Einsatz einer Fremdsprache helfen werden. Sie hatten erfahren, dass sie selbst und auch alle andern Teilnehmer/innen in ihren Gruppen einen zunächst fremden, schwierig erscheinenden Text entschlüsselt und ihn sich erarbeitet hatten. Besonders die lyrischen Texte $^{10}$, die den Schüler/innen zunächst den Zugang zu verweigern schienen, hatten intensive Prozesse der Aneignung und der eigenen Bearbeitungen initiiert. Auf der Grundlage des vorangegangenen Arbeitsprozesses konnten alle Beteiligten ihre Gruppenergebnisse in deutscher Sprache präsentieren.

Dass der fächerübergreifende Ansatz eineAuseinandersetzung mit historischen Ereignissen und Abläufen bewirkt hatte, war in allen Präsentationen zu sehen. Die Themen Fremdenfeindlichkeit in Südafrika, Rassismus in einer Schule kurz nach Ende der Apartheid, das Pro und Contra einer Flucht aus der DDR in den Westen waren von den Schüler/innen in einen jeweils ganz persönlichen Rahmen eingebettet worden. Voraussetzung dafür war (ausgehend von den zu Grunde liegenden Texten) die Erarbeitung eines gemeinsamen Verständnisses, eines Sich-Hineinfühlens durch dramapädagogische Übungen und Arbeitsformen wie die 'Frage des Tages'.

Über den mehrtägigen Workshop hinaus setzten wir das Projekt fort: eine Dokumentation unserer Arbeit und der Ergebnisse sollte entstehen und Lehrer/innen und Schüler/innen zur Verfügung gestellt werden. ${ }^{11}$ Ein Redaktionsteam wurdevon den Teilnehmer/innen gewählt, das sich nach Verlauf einiger Wochen traf. Die Schüler/innen brachten Fotos und selbst geschriebene Texte mit, die ihre weitere Auseinandersetzung mit der Projektthematik illustrierten. Die Identifikation mit Handeln, Leiden und Aufbegehren von Menschen in der Vergangenheit zeigt dies Beispiel: die Jugendlichen hatten auf den Film „Skin“12 und dessen authentische Geschichte sehr emotional reagiert, sie wollten daher eine Seite für die Dokumentation dazu gestalten. Es fiel ihnen

\footnotetext{
${ }^{10}$ Lebogang, Mashile (2010): Töchter von morgen - Gedichte. Heidelberg, Das Wunderhorn $\mathrm{GmbH}$.

${ }^{11}$ Die Publikation Literatur und Geschichte - und wir hat für südafrikanische Deutschlehrende besondere Bedeutung, weil sehr wenige Materialien für den Unterricht zur Verfügung stehen; die Mittel für die Anschaffung von Lehrwerken sind äußerst begrenzt und den europäischen Fremdsprachen wird seitens des Ministeriums vergleichsweise geringe Relevanz zuerkannt.

12 Der Film ist als DVD erhältlich. Er erzählt die Geschichte der Südafrikanerin Sandra Laing, die als Kind weißer Eltern aufgrund der Apartheidsgesetze als „,coloured“ eingestuft wird.
} 
allerdings schwer, ihre Gedanken und Empfindungen in Worte zu fassen - diese blieben recht klischeehaft. Es gelang ihnen aber, andere Ausdrucksmittel für die eigene Betroffenheit zu finden, so die Zeichnung einer stilisierten Filmrolle, die in Einzelbildern die Gesichter der Projektteilnehmer/innen zeigt (siehe Anhang).

Aufgrund der verschiedenartigen Aufgabenstellungen in der Großgruppe und der komplexen Anforderung einer Präsentation hatten alle Jugendlichen die Möglichkeit gehabt, sich mit ihren jeweiligen Fähigkeiten einzubringen. Die Vernetzung der Schulen über die beteiligten Personen, Schüler/innen und Lehrer/innen wurde in Gang gesetzt. Die Redaktionssitzung, die einige Wochen nach dem Projekt stattfand, war ein weiterer Schritt dahin: auch über das so entstandene Buch setzen sich die Beteiligten weiter auseinander. Eine Nachhaltigkeit wird dann entstehen, wenn weitere schulübergreifende Ereignisse diese Anfänge fortführen.

Ein Prozess des Sich-Erinnerns an eigene Erlebnisse setzte auch bei den Lehrer/innen ein, der Wunsch darüber zu berichten und sich auszutauschen machte deutlich, dass der Verlauf der Geschichte eben keineswegs von uns als Individuen getrennt werden kann. Lehrkräfte als Zeitzeugen - etwa als eine der ersten, die am 11. November 1989 von Ost- nach Westberlin durchs Brandenburger Tor ging - konnten eine Gesprächsebene mit Schüler/innen finden, auf der sie als ganz persönliche Experten erzählten, berichteten und befragt wurden. Umgekehrt erwiesen sich auch Jugendliche gegenüber ihren Lehrer/innen und anderen Jugendlichen als Experten. Über die vertrauensvolle Zusammenarbeit, das konstruktive, kritische und weiterführende Gespräch der Lehrkräfte und der Projektleiterin ist ein weiteres Netz entstanden.

Einige Schüler/innen äußerten in der abschließenden Evaluation des Projekts ihre Zufriedenheit damit, die Bedeutung des konstruktiven Feedback im Gegensatz zu pauschaler, klischeehafter oder persönlicher Kritik erkannt und diese Methode erfahren und angewandt zu haben.

\section{Was machte das Projekt zu einem Praxislabor?}

Die Projektleitung unterbreitete ein Angebot ausgehend von Wünschen und Bedürfnissen der teilnehmenden Gruppe. Hier war es der Wunsch nach einer schulübergreifenden Zusammenarbeit von Schüler/innen, die sich für Jugendliteratur im Fach Deutsch interessieren.

- Die Thematik - Literatur und Geschichte - nahm Bezug auf individuelle, biographische Erfahrungen und Kenntnisse und verknüpfte sie mit relevanten gesellschaftlichen Fragestellungen.

- Die Teilnehmer/innen brachten ihre Erfahrungen, Kenntnisse, Ideen so in das Projekt ein, dass sie den Verlauf und Prozess mit steuerten.

- Die Verschiedenheit der Einzelnen bereicherte den Prozess und alle Teilnehmenden: die verschiedenen Sprachen etwa wurden wahrgenommen 
und förderten die Sprachbewusstheit aller.

- Der Prozess der Arbeit im Projekt und ihr Ergebnis war offen - jede Arbeitsgruppe aber präsentierte abschließend ihr Ergebnis.

- Lehrende waren während des Projekts Teilnehmer/innen und Berater/innen. Team-Besprechungen waren auch Schritte zu späterer Kooperation, boten Möglichkeiten gegenseitiger pädagogischer und fachlicher Weiterbildung.

- Szenische Verfahren, Körperarbeit und fächerübergreifendes thematisches Arbeiten ergänzten sich produktiv.

Ausgehend von den Erfahrungen in diesem Projekt und den Projekten "Sprachfluss" sowie „The Climate is Changing“"13 entwickelten Edda Holl und ich das Format der Praxislabors. Dies sieht ausdrücklich vor, dass Teams für die Planung und Durchführung der Praxislabors verantwortlich sein sollten. Diese Teams setzen sich zusammen aus erfahrenen (Theater-)Pädagogen, die „Von außen" in Schulen und andere Institutionen der Bildungsarbeit kommen können.

\section{Einsatz der „PROBE!“-Teams}

Praxislabors von,,Probe!“-Teams können an verschiedenen Orten stattfinden. Die gegenwärtige Analyse der Bildungslandschaft und die Diskussion um Innovation und Veränderung in der Schule hat die Notwendigkeit von Umstrukturierungen sowohl in der Organisation - z.B. Ganztagsangebote, Fördermaßnahmen, jahrgangsübergreifender Unterricht - wie auch hinsichtlich der Unterrichtsentwicklung - z.B. kooperatives und selbstgesteuertes Lernen, schüleraktivierende Maßnahmen, interdisziplinäre Ansätze - aufgezeigt. Hinsichtlich solcher Desiderata wird deutlich, dass kein Erkenntnisproblem besteht, hingegen es aufgrund vieler Sachzwänge Umsetzungsprobleme in den Institutionen gibt. In dieser Situation machen die „PROBE!“-Teams ein Angebot. Sie unterstützen die Institutionen hinsichtlich Konzeption, Organisation, Durchführung und Evaluierung von künstlerischen Projekten. Sie tun dies auf der Grundlage von Bedürfnissen und Wünschen der jeweiligen ,Auftraggeber' in Schulen oder Seminaren.

\subsection{Schule}

In der Schule sehen sich Lehrkräfte Situationen in ihrem Unterricht gegenüber, die innerhalb der Schultage mit ihrem 45-Minuten-Takt nicht immer aufzufangen sind und daher nicht bearbeitet werden können. Die Vielfalt der

\footnotetext{
13 Die Projekte wurden aus Mitteln der PASCH-Initiative vom Goethe-Institut in SubsaharaAfrika und Deutschland finanziert, konzipiert und geleitet von Edda Holl.
} 
Religionen, kultureller Verhaltensregeln, der Sprachen wird in den Schulen selten als Chance wahrgenommen, vielmehr als ein Problem bei gemeinsamen Unternehmungen, im Sport, ganz besonders aber, wenn das Erreichen von Regelstandards für Schüler/innen mit nicht-deutschsprachigem Hintergrund schwierig wird. Dabei möchte ich Heterogenität in Lerngruppen keineswegs auf Verschiedenheit von Sprachen und Kulturen begrenzt definieren: Vorwissen, sozialer Hintergrund, Interessen und Kompetenzen sind unterschiedlich. Die Erziehungswissenschaftlerin von der Groeben fordert, alle Verschiedenheit zu nutzen:

Inklusive Systeme zeigen, dass und wie es möglich ist, alle Kinder und Jugendlichen ,mitzunehmen', exklusive setzen auf homogene Leistungsgruppen. Das können sie tun, weil für die Vergabe von Noten, Abschlüssen, Berechtigungen (leider) nicht maßgebend ist, ob und wie viel ,Lust auf die Begegnung mit der Welt' die Schule den Heranwachsenden mitgegeben hat, sondern allein das dabei erworbene Wissen. (von der Groeben 2008: 38)

In einem Unterricht, der von einer gleichermaßen vollständigen Beherrschung der Unterrichtssprache ausgeht, entstehen für Nicht-Muttersprachler Unsicherheit und Motivationsverlust, die muttersprachlichen Schüler/innen erleben Langeweile, ablehnende Gefühle gegenüber den ,anderen', von denen sie sich aufgehalten fühlen. Unterrichtsstörungen, destruktive Gruppenbildungen innerhalb der Lerngruppe und fehlender Lernerfolg können in der Folge entstehen. Lehrkräfte sollen in dieser Situation das Unmögliche möglich machen: individuell fördern und auf standardisierte Leistungsabfragen vorbereiten. Da Praxislabors sowohl auf Mehrsprachigkeit als auch auf kreative Talente der Einzelnen setzen, können sie dieser Situation anders begegnen und dem in der Schule geforderten und so oft nicht erfolgreichen Spagat von individuellem Fördern und standardisierten Tests andere Möglichkeiten des Lernens entgegen setzen. Ihr fächerübergreifender Ansatz, die Aufgabenstellungen und die Lernumgebung bieten den Teilnehmenden vielfältige Lernzugänge. Lernerfolge zeigen sich in den abschließenden Präsentationen und auch in der Dokumentation des Projekts: Schülerleistungen, die aus dem Projekt hervor gegangen sind, aber nicht im Vorweg geplant werden konnten. Die Praxislabors leisten daher einen Beitrag zur Leistungssteigerung im Fach - gerade weil sie anders verfahren als der durch vielfache Regeln bestimmte schulische Unterricht.

Praxislabors fördern mit Verfahren, die Spracharbeit und Theaterübungen verbinden, Sprach(en)bewusstheit und setzen auf das Lernen von Sprachen auf neuen, kreativen Wegen. Sie erfüllen damit Forderungen, wie sie von Hufeisen an ein Gesamtsprachencurriculum gestellt werden. Es geht darum,

die vorhandene(n) individuelle(n) Mehrsprachigkeit(en) der Lernenden, die sie bereits mit in den institutionellen Kontext mitbringen, zu berücksichtigen und in den schulischen Alltag mit einzubeziehen [...], die geplanten schulischen Mehrsprachigkeit(en) zu fördern, die Lehrenden [...] für Fragen rund um Mehrsprachigkeit, das multiple Sprachenlernen, Mehrsprachigkeitsdidaktik und das Sprachenlernen überhaupt zu 
sensibilisieren [... und] Interkulturelles in alle Fächer einzubinden [...]

(Hufeisen 2011: 266f)

Die Praxislabors fördern durch ihre Verfahren ebenfalls einen Prozess des Team-building in der Gruppe. Die abschließende Präsentation eines Workshops gelingt dann, wenn alle Einzelnen sich als Teil des Ensembles fühlen. Diesen Prozess initiieren die „PROBE!“-Teams. Auf einer solchen Erfahrung kann die Lerngruppe in der Folgezeit aufbauen.

Im Verlauf eines Schuljahrs gibt es verschiedene Eckdaten und Ereignisse, an denen die Durchführung eines Praxislabors sinnvollerscheint wiebeispielsweise, wenn Lerngruppen beim Übergang in die weiterführende Schule oder etwa im Zusammenhang mit der Sprachenwahl neu zusammengesetzt werden . An dieser Stelle wäre ein Einstieg sinnvoll, der von der Mehrsprachigkeit innerhalb der Gruppe ausgeht. Auf diese Weise kann die Sprachenbewusstheit gefördert und damit ein Schritt zu einer schülerorientierten Lernökonomie getan werden.

Besonders wenn sich die Schülerinnen und Schüler noch gar nicht kennen, wird so auch ein Gruppengefühl entwickelt, das sich in der Unterrichtsarbeit positiv auswirkt.

Projekttage zum Thema Sprachenvielfalt in der Schule oder in der Klasse: hier ist darauf zu achten, alle Kinder und Jugendlichen in ihrer Komplexität wahrzunehmen und anzuerkennen, ihre Individualität nicht auf ihre Wurzeln in einer anderen Kultur und Sprache als der deutschen zu reduzieren, die deutschen Kinder und Jugendlichen nicht als „weniger exotisch“ dastehen zu lassen.

Klassenfahrten werden für Lehrerinnen und Lehrer oft eine besondere Herausforderung, denn dort gilt es die Jugendlichen ,rund um die Uhr' zu gemeinsamen Unternehmungen zu motivieren. Die ,attraktiven Ziele' solcher Fahrten - Skifreizeiten, Fahrten ans Meer - können aufgrund finanzieller Belastung der Familien nicht immer angesteuert werden. Es steht ausreichend Zeit zur Verfügung, in diesem Rahmen ein an den Bedürfnissen der Lerngruppe anknüpfendes Theaterprojekt zu gestalten, das anschließend der Schulöffentlichkeit präsentiert wird.

Im Rahmen der Ganztagsschule kann - möglicherweise in einem Nachmittagsangebot - über einen längeren Zeitraum hinweg an der Thematik der Mehrsprachigkeit, kultureller Vielfalt gearbeitet werden, wobei auch jahrgangsübergreifende Gruppen zu einem Ensemble zusammengeführt werden können.

\subsection{Lehrerausbildung}

Im Zusammenhang mit Seminaren zu Didaktik und Methodik des Fremdsprachenunterrichts, des muttersprachlichen Unterrichts und der Pädagogik stellen „PROBE!“-Teams ihre Arbeitsweise vor dem Hintergrund der Mehrsprachigkeitsdidaktik, der Unterrichtsentwicklung (schüleraktivierende Lernformen, handlungsorientierter Unterricht, kooperatives Lernen, Lernen mit allen Sinnen) in Wochenend- oder Tagesveranstaltungen vor. 


\subsection{Künstlerische Studiengänge}

Absolventen kunstpraktischer Studiengänge verfügen über Wissen und Erfahrungen mit künstlerischen Lern- und Produktionsprozessen. Besonders Kulturschaffende aus dem Bereich der ,sozialen Kunstform' Theater, Schauspielerinnen und Schauspieler, Regisseurinnen und Regisseure sowie Theaterpädagoginnen und -pädagogen sind mit kollektiven Arbeitsprozessen vertraut. Ihre Kompetenzen in Sachen Gruppen- oder Ensemblebildung, Konzeption und Medialisierung von literarischen und alltäglichen Stoffen, Präsentationsformen, Sprech- und Probentechniken können schulischen Unterricht bereichern und ergänzen. Die „Probe!“-Teams wollen eine Lücke schließen, indem sie schulische Projekte zusammen mit Gruppen theaterpraktischer Studiengänge und Lehrkräften erforschen und erproben. In solchen Praxislabors kann erfahren werden, in welcher Weise sich theaterpraktische Arbeitsformen sinnvoll und gewinnbringend im Rahmen von Schule einsetzen lassen.

\subsection{Institutionen der Weiterbildung}

Der Anspruch des ,lebenslangen Lernens' bedeutet auch, dass nach Abschluss der eigenen Ausbildung Angebote von Institutionen der Kommunen, Universitäten, Gewerkschaften und anderer Körperschaften zur Verfügung stehen. In diesem Kontext möchten „Probe!“-Teams anbieten, Lehrerinnen und Lehrern kreative, aktivierende und motivierende Arbeitsformen für den Unterricht vorzustellen.

\section{Fazit}

In der Diskussion um die Innovation von schulischen Lernprozessen leisten die „PROBE!“-Workshops einen weiterführenden Beitrag zu neuen Lernwegen und Unterrichtsentwicklung. Die Praxislabors gehen in ihren Verfahren selbstverständlich von einer Heterogenität der Teilnehmergruppe aus. Diese Heterogenität wird produktiv gemacht hinsichtlich der vorgefundenen Mehrsprachigkeit, der individuellen Lernstärken und Kompetenzen, der Interessen an Zugängen zu einem Thema. Die Teilnehmer/innen an den Praxislabors können aufgrund der Komplexität und Vielfalt der Aufgaben ihre Begabungen und Fähigkeiten entfalten. Übungen aus der Theaterarbeit ermöglichen neue Wahrnehmungsweisen des Themas, der Einzelnen und der Gruppe, des Ausprobierens von individuellen Ausdrucksformen. Im Prozess der gemeinschaftlichen Erstellung einer Präsentation erleben sich die Kinder und Jugendliche als Verantwortliche und als Expert/innen. Die „PROBE!“-Teams unterstützen die Lehrkräfte in ihrem Bemühen, die Lerngruppen zu wirklichen Ensembles zusammenzuführen und auf dieser Grundlage kooperative und selbstgesteuerte Lernprozesse zu fördern. Aus diesem Grunde stellen die Praxislabors eine vielversprechende Lehr- und Lernform auf dem Weg zu einer Schule der Vielfalt 
dar. $^{14}$

\section{Bibliographie}

Fasse, Gisela (2010): Literatur und Geschichte - und wir. Ein schul- und fächerübergreifendes Projekt in Südafrika. http://www.auslandsschulwesen.de/Auslandsschulwesen/Auslandsschularbeit/Fachberater/Afrika 6.1.2012. Siehe auch: http://www . kreative-lernwege.com, 6.1.2012

Holl, Edda (2011): SPRACH-FLUSS, Theaterübungen für Sprachunterricht und interkulturelles Lernen. Ismaning: Hueber

Hufeisen, Britta (2011): Gesamtsprachencurriculum: Weitere Überlegungen zu einem prototypischen Modell. In: Baur, Rupprecht S. und Hufeisen, Britta: „Vieles ist sehr ähnlich“. Individuelle und gesellschaftliche Mehrsprachigkeit als bildungspolitische Aufgabe. Hohengehren: Schneider, 265-282

Lebogang, Mashile (2010): Töchter von morgen - Gedichte. Heidelberg: Das Wunderhorn $\mathrm{GmbH}$

Mecheril, Paul (o.J.): Anerkennung des Andern als Leitperspektive interkultureller Pädagogik? Perspektiven und Paradoxien.

http://www.ida-nrw.de/projekte-interkulturell-

nrw/such_ja/12down_1/pdf/mecheril.pdf, 6.1.2012

Terkessidis, Mark (2010): Interkultur. Berlin: Suhrkamp

von der Groeben, Annemarie (2008): Verschiedenheit nutzen. Besser lernen in heterogenen Gruppen. Berlin: Cornelsen

${ }^{14}$ Der Begriff erscheint bei von der Groeben (2008: 184ff), nach der die „Schule der Vielfalt“ sich über ihren Umgang mit Heterogenität. 


\section{Anhang}

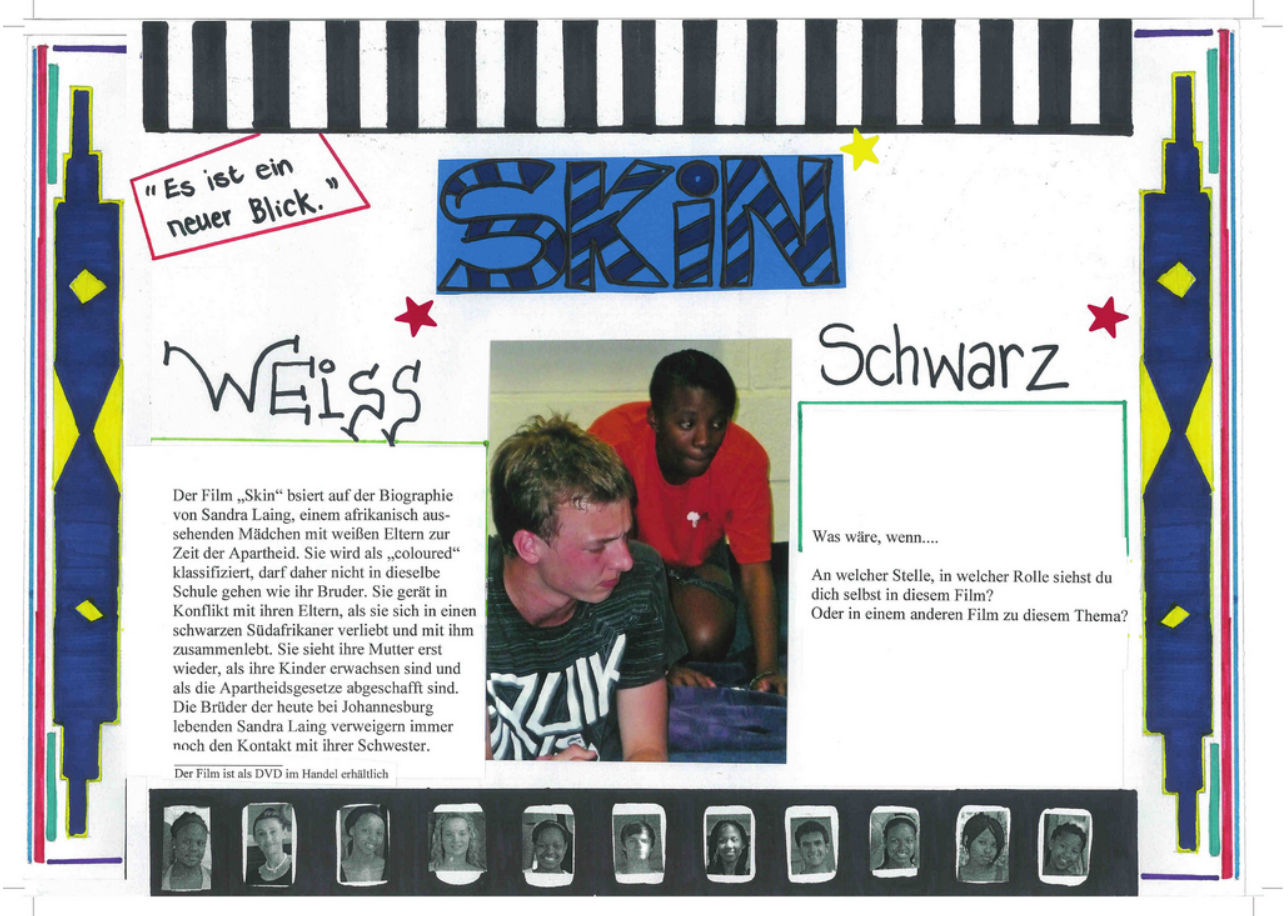

Abbildung 1: Stilisierte Filmrolle.

Hier eine Schülerarbeit zum Spielfilm „Skin“. Die Schülerinnen und Schüler setzten Fotos, die während der Projekttage entstanden waren, in eine selbst gezeichnete Filmrolle ein. Die Arbeit entstand während der Redaktionssitzung in Pretoria, einige Wochen nach den Projekttagen (siehe Fasse 2010). 